\title{
FUZZY REASONING TO MORE ACCURATELY DETERMINE VOID AREAS ON OPTICAL MICROGRAPHS OF COMPOSITE STRUCTURES
}

\author{
JESUS A. DOMINGUEZ \\ QinetiQ North America Inc. \\ Kennedy Space Center, Mail Stop ESC-58, Florida 32899 \\ Jesus.a.dominguez@nasa.gov \\ LANETRA C. TATE \\ NASA \\ Kennedy Space Center, Mail Stop NE-L4, Florida 32899 \\ lanetra.c.tate@nasa.gov \\ M. CLARA WRIGHT \\ NASA \\ Kennedy Space Center, Mail Stop NE-L1, Florida 32899 \\ m.clara.wright@nasa.gov \\ ANNE CARACCIO \\ NASA \\ Kennedy Space Center, Mail Stop NE-L6, Florida 32899 \\ anne.caraccio@nasa.gov
}

\begin{abstract}
Accomplishing the best-performing composite matrix (resin) requires that not only the processing method but also the cure cycle generate low-void-content structures. If voids are present, the performance of the composite matrix will be significantly reduced. This is usually noticed by significant reductions in matrix-dominated properties, such as compression and shear strength. Voids in composite materials are areas that are absent of the composite components: matrix and fibers. The characteristics of the voids and their accurate estimation are critical to determine for high performance composite structures. One widely used method of performing void analysis on a composite structure sample is acquiring optical micrographs or Scanning Electron Microscope (SEM) images of lateral sides of the sample and retrieving the void areas within the micrographs/images using an image analysis technique. Segmentation for the retrieval and subsequent computation of void areas within the micrographs/images is challenging as the gray-scaled values of the void areas are close to the gray-scaled values of the matrix leading to the need of manually performing the segmentation based on the histogram of the micrographs/images to retrieve the void areas. The use of an algorithm developed by NASA and based on Fuzzy Reasoning (FR) proved to overcome the difficulty of suitably differentiate void and matrix image areas with similar gray-scaled values leading not only to a more accurate estimation of void areas on composite matrix micrographs but also to a faster void analysis process as the algorithm is fully autonomous.
\end{abstract}

Key Words: Composite Void Analysis; Optical Micrographs Analysis; Void Content Composites; Void Content Resin; Void Content Matrix; Fuzzy Reasoning; Fuzzy Logic; Image Segmentation; Image Binarization; Binary Segmentation

\section{INTRODUCTION}

In this paper we illustrate the advantages of using a FR-based imaging technique developed and patented by NASA [1][2] to autonomously perform binary image segmentation on optical micrographs and SEM images of composite samples and more accurately extract the void areas. This FR-based technique has proven its capability of distinguishing contiguous areas with similar gray-scaled values as it often happens with void and matrix areas within optical micrographs and SEM images of composite samples [3]. The technique applies two complementary image-analysis algorithms, one named FRIEB (Fuzzy Reasoning Image Enhancement Binarization) that enhances the image for binarization (binary segmentation), and the second named FRAT (Fuzzy Reasoning Adaptive Thresholding) that 
performs the binary segmentation of the image enhanced via FREIB. Both algorithms, FREB and FRAT are based on FR approach and were patented by NASA [1][2].

\section{FUZZY REASONING (FR) IMAGE TECHNIQUES}

The new complementary image processing algorithms FREIB and FRAT extract information of an image in a binary/Boolean form being one the image background and zero the image foreground utilizing FR-based reasoning to handle the uncertainty, imprecision, and incompleteness naturally involved in the binary segmentation process of grayscaled/color images [4]. FR work's aim is to tolerate approximate reasoning and partial truth in order to achieve tractability, robustness, low solution cost, and close resemble with human like decision making on gray-scaled/color images analysis. The system provides sufficient provision for representing the uncertainties at the gray-scaled image so the enhancement (FREIB) and subsequent binary segmentation (FRAT) of the image can be associated with less uncertainty and better performance [5].

\subsection{Fuzzy Reasoning Image Enhancement for Binary Segmentation (FRIEB)}

One of the key goals of performing binary segmentation of grayed-scaled images is to be able to digitize text, numbers, and the location of objects or regions of interest contained in grayed-scale image acquired having uncontrolled image acquisition conditions (lighting, camera/video stabilization, etc.). These conditions degrade the ending quality of the acquired gray-scaled image making it more difficult to binarize the text, numbers, and objects of interest contained in the image. In optical micrographs and SEM images of composite samples, the image acquisition process is fully controlled and quality of the image is not an issue, but similarity in gray-scale values of the region of interest (void areas) and the matrix makes existing conventional binary segmentation algorithms unfeasible or unreliable. FRIEB uses fuzzy reasoning based on heuristic techniques that compare the intensity value of each pixel in the image with the intensity levels of the immediate neighbors and decide if the intensity value of the pixel needs to be reduced or increased. A detailed description of FRIEB algorithm is found in the patent document filed by NASA [1].

\subsection{Fuzzy Reasoning Adaptive Thresholding (FRAT)}

Binary image processing is of special interest because an image with binary format can be processed with very fast logical (Boolean) operators. Since most image display systems and software assume images of 8 or more bits per pixel, the binary segmentation of these images usually takes 2 extreme gray tones, black and white, which are ordinarily represented by 0 or 255 , respectively, in an 8-bit gray-scale display environment. Image thresholding is the simplest image segmentation approach. It is actually a pattern classification procedure in which only one input feature is involved, the pixel intensity value. The key is to automatically choose an optimal threshold value so that the number of misclassified image pixels is kept as low as possible. Obviously, the threshold that is chosen has a critical importance since it controls the binary-based pattern classification that is obtained from the gray-level image.

In FRAT, binary threshold is chosen assuming that the image is an array of singletons corresponding to image pixels, each having a membership value associated with a property of the pixel, the grayness level. For image thresholding, the membership function is defined in terms of the grade of pixel's grayness belonging to one of the two classes, background and foreground. The membership function is calculated via a triangular function at each of the two classes (background and foreground); the triangular function in each of the two classes is built using the average image grayness and histogram-based weight values at each class. In each of the two classes, the membership value (equal to 1) is the largest at the class average gray level and reduces its value when the difference between the pixel gray level and its class average level increases. This means that pixels with gray levels close to their corresponding class average gray levels have less fuzziness or ambiguity and thus can be classified with greater confidence than pixels with gray levels far from their class gray levels. The image entropy measure is used as a cost function to find the optimal threshold. The entropy factor needed to compute the entropy measure is calculated using a simple and fast computational linear function. A detailed description of FRAT algorithm is found in the patent document filed by NASA [2].

\section{VOID DETERMINATION EXAMPLES}

\subsection{Optical micrograph of Carbon Fiber Unitapes}

Figure 1 illustrates a lateral-sided optical micrograph of a composite sample manufactured at the Kennedy Space Center (KSC) using eight prepreg plies of CYCOM HR40/5320-1 carbon fiber unitapes with [0/45/90/-45]s layup; in the image white segments correspond to the carbon fiber material, dark black segments correspond to void cavities, and dark gray areas correspond to the matrix.

Image segmentation for the retrieval and subsequent computation of void areas is challenging as the gray-scaled values of the void segments are close to the gray-scaled values of the matrix. Figure 2 shows the histograms of the three primary colors (Red, Green, and Blue) of the micrograph of figure 1. According to these three histograms shown 
in figure 2, most of the void and matrix occurrences happen to be within the dark gray segment region making it difficult to separate them when both matrix and void segments are contiguous.
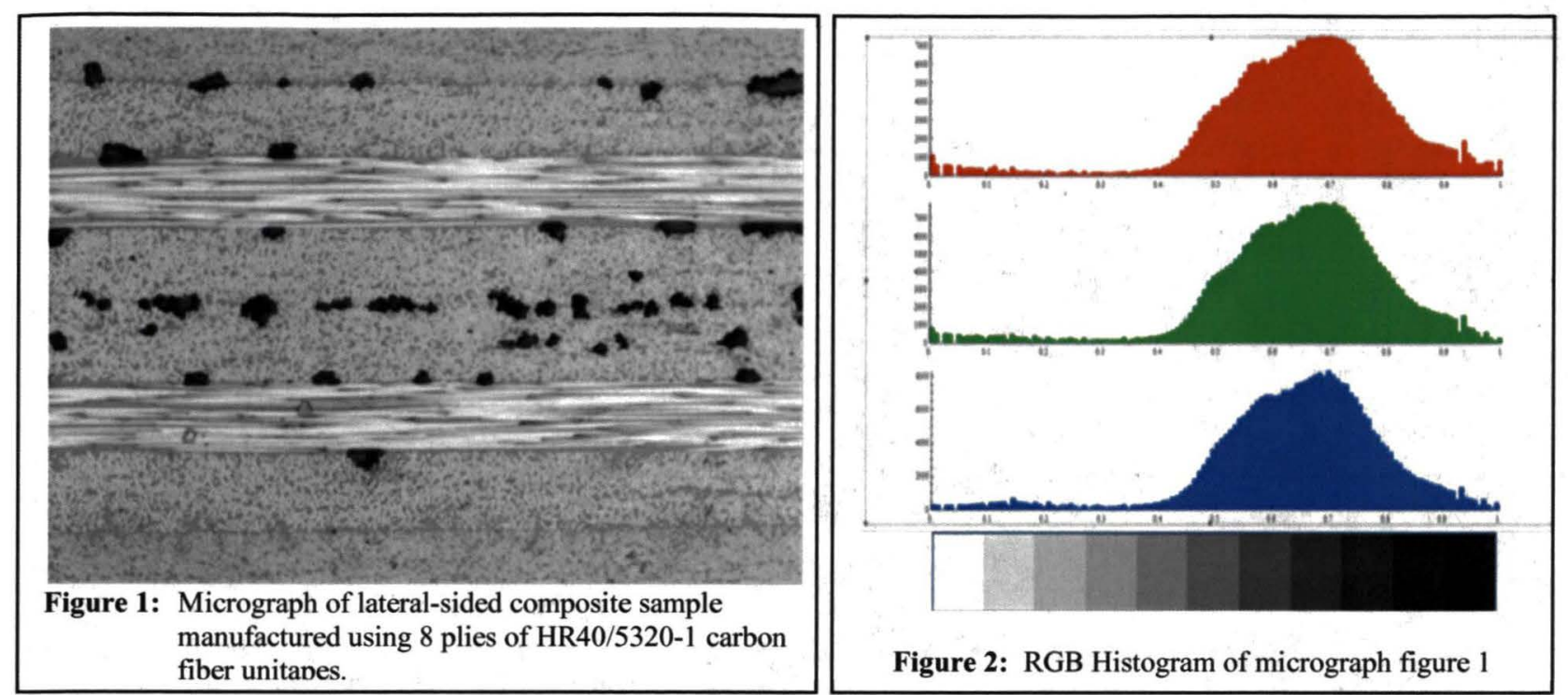

Optimal binary segmentation performed on the optical micrograph shown in figure 1 using commercially available image analysis software tools, such as Mathematica ${ }^{\mathrm{TM}}$ yields the binary segmentation displayed in figure 3 . Figure $4 \mathrm{~A}$ shows a cropped section of figure 3 having two areas with contiguous void and matrix segments; figure 4B shows the corresponding binary segmentation obtained via Mathematica ${ }^{\mathrm{TM}}$; figure $4 \mathrm{C}$ indicates in red the two segments assigned as void segments. It is clear that Mathematica ${ }^{\mathrm{TM}}$ binary segmentation algorithm cannot differentiate two contiguous matrix and void segments present in figure $4 \mathrm{~A}$ assigning both segments as a single void segment in both cases.

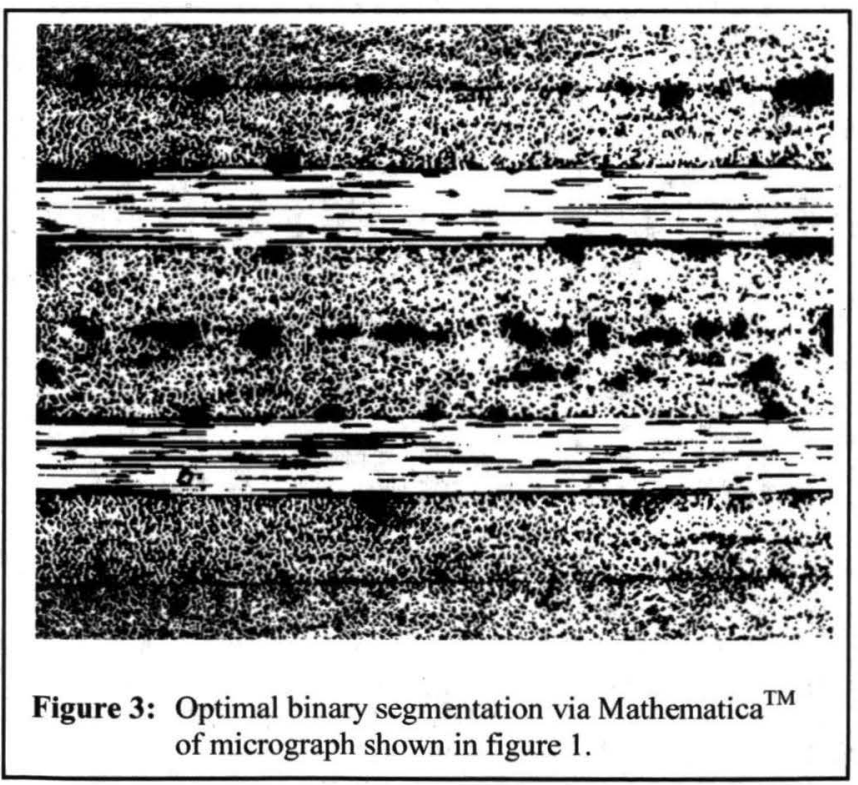

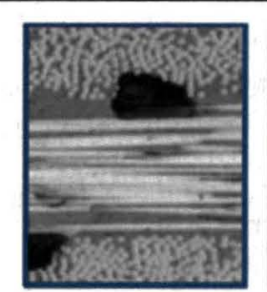

A

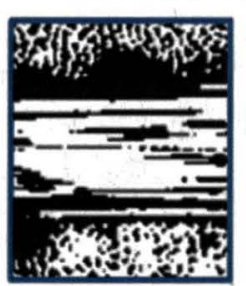

B

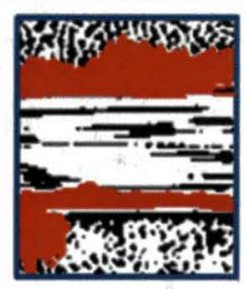

C

Figure 4: Binary segmentation of micrograph shown in figure 1 via Mathematica ${ }^{\mathrm{TM}}$ (figure 3) assigns contiguous matrix and void segments as a single void segment.

A Cropped section of micrograph (figure 1)

B Binary segmentation via Mathematica ${ }^{\mathrm{TM}}$ of the micrograph (cropped section A)

C Areas in red assigned as void segments by binary segmentation via Mathematica ${ }^{\mathrm{TM}}$

Segmentation performed manually by using two thresholds (three sections) on the histograms shown in figure 2 instead of one threshold (as in binary segmentation of figure 3 ) was able to differentiate and separate contiguous matrix and void segments within the micrograph but concurrently it wrongly assigns some matrix segments as void segments. Figure 5 shows the segmentation manually performed on the micrograph shown in figure 1 using two thresholds instead of one as used in binary segmentation. Figure 6 displays the cropped area of figure 5 showing the correct separation of matrix and void segments that Mathematica ${ }^{\mathrm{TM}}$ fails to separate (as illustrated in figure 4) but at 
the same time this manual two-threshold segmentation algorithm wrongly assigns a matrix segment as a void segment as illustrated in figures $6 \mathrm{~B}$ and $6 \mathrm{C}$.

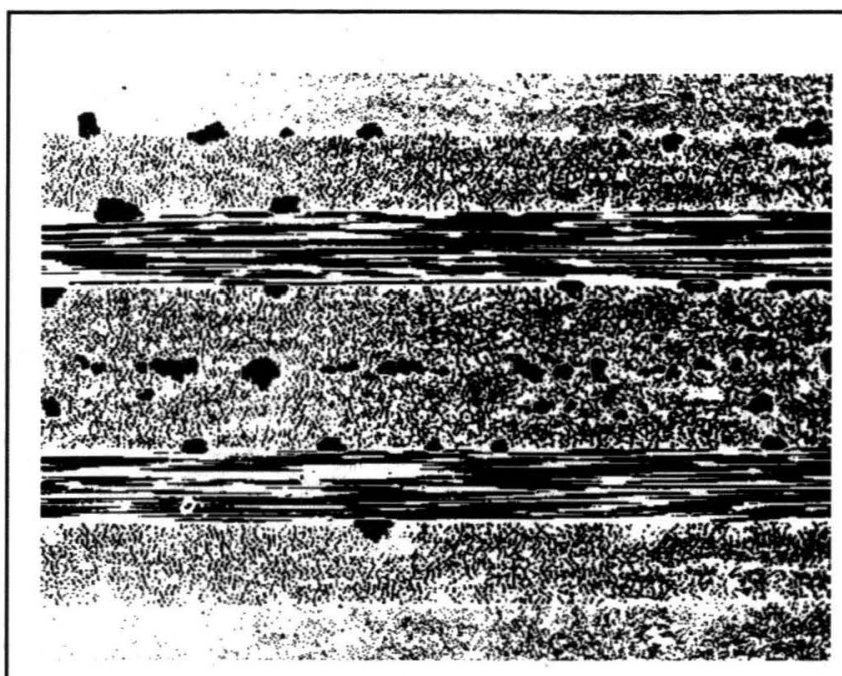

Figure 5: Binary segmentation of figure 1 using three sections

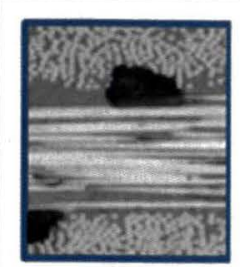

A

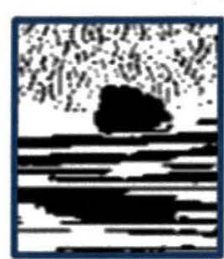

B

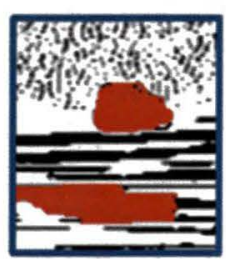

C

Figure 6: Segmentation of micrograph shown in figure 1 using two thresholds (figure 5) differentiates matrix and void segments but at the same time assigns some resin segments as void segments. A Cropped section of the micrograph (figure 1) B Manual segmentation via 2 thresholds of the micrograph shown in the cropped section A. C Areas in red assigned as void segments by manual 2-threshold segmentation.

Standard image analysis methods used at KSC fails to differentiate in some cases matrix and void segments yielding void areas larger than actual ones as illustrated in figures 3 and 4. Manual tuning of the standard method helps correct and differentiate these two segments but starts assigning matrix areas as void areas as illustrated in figures 5 and 6 .

The image analysis technique based on Fuzzy Reasoning (FR) developed at KSC [1][2][3] and described above was successfully used to automatically determine the void areas. Figures 7 and 8 illustrate the binary segmentation of the micrograph shown in figure 1 . This technique shows clearly accurate determination and retrieval of void segments as shown in the cropped images of figure 8.

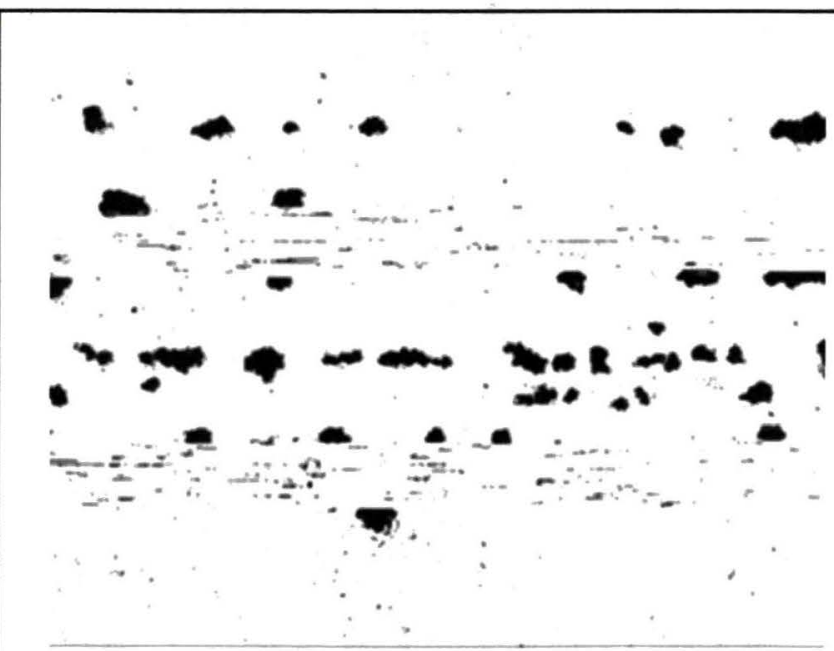

Figure 7: Binary segmentation of figure 1 using new technique based on Fuzzy Reasoning (FR) approach

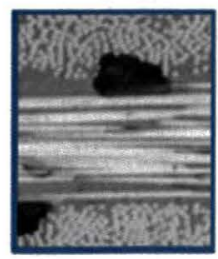

A

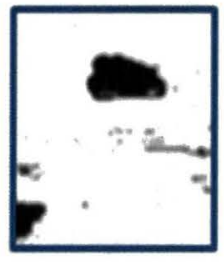

B

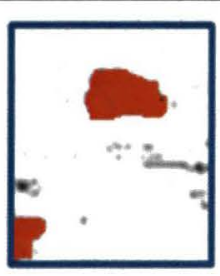

C
Figure 8: Binary segmentation of figure 1 using new technique (figure 7) correctly differentiates matrix and void segments

A Cropped section of the micrograph (figure 1) B FR-based binary segmentation of the micrograph shown in the cropped section A. C Areas in red assigned as void segments by FRbased binary segmentation 
A final noise reduction (using standard methods) of the binary image originally generated after performing binarization enhancement via FREIB and binary segmentation via FRAT leads to a final binary image of figure 9 that shows the void segments as black blobs. Total void fraction is straightforward calculated from figure 9 (binary image) by computing the areas of all black blobs (void segments) and dividing it by the total image area.

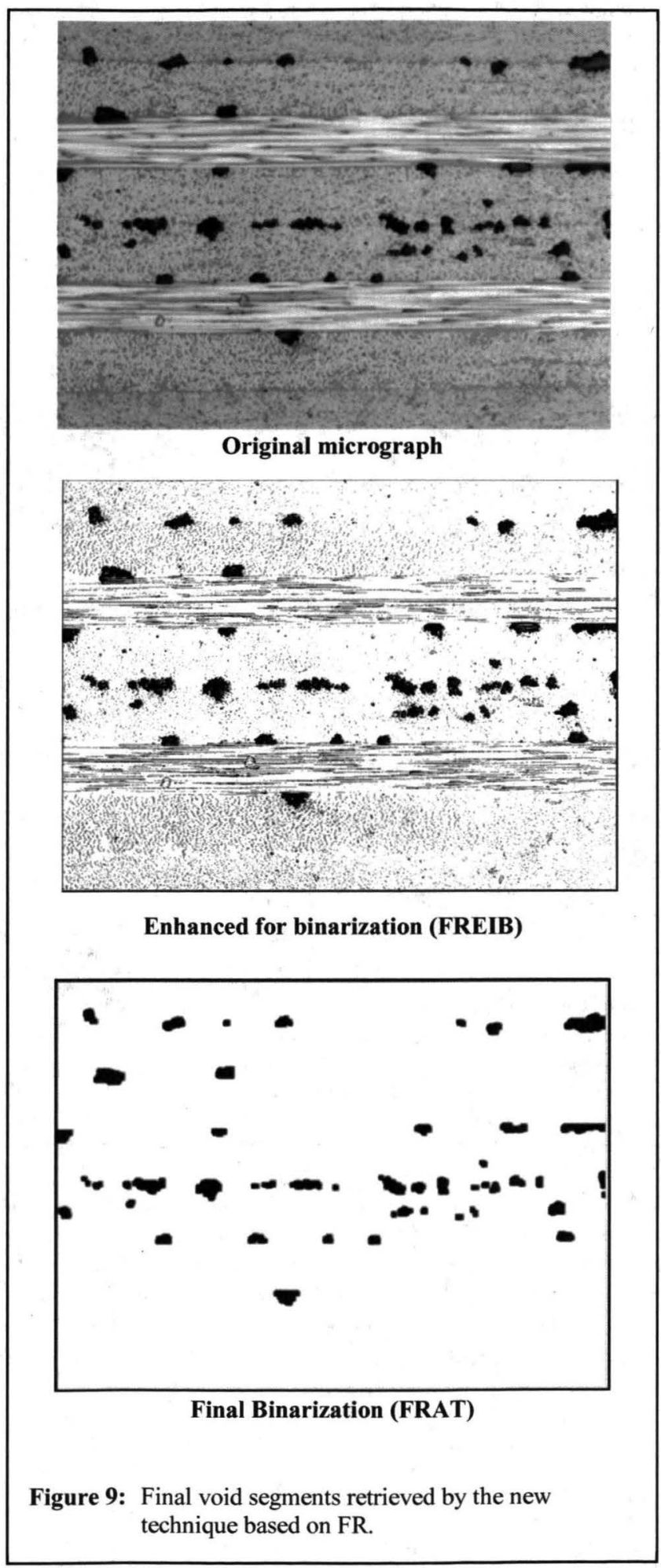




\subsection{SEM Image of Hybrid Composites}

An alternative to carbon fiber prepregs is to combine boron fibers with graphite prepreg in an epoxy matrix to produce high-performance materials with exceptional properties. By altering the spacing of the boron fibers in the prepreg, performance properties and material costs can be tailored to meet the needs of a variety of demanding composite applications. Figure 10 shows a SEM image acquired on 4-ply composite panel fabricated using 2 plies of CYCOM HR40/5320-1 and 2 plies of Hybor ${ }^{\circledR}$ boron/carbon fiber blend. Table 1 displays the layup of this 4-ply composite panel.

\begin{tabular}{|c|c|c|}
\hline Ply & Orientation & Type \\
\hline 1 & 0 & Hy-Bor \\
\hline 2 & 90 & HR40/5320-1 \\
\hline 3 & 90 & HR40/5320-1 \\
\hline 4 & 0 & Hy-Bor \\
\hline
\end{tabular}

Table 1 4-ply composite panel using HR40/5320-1 and Hybor ${ }^{\circledR}$

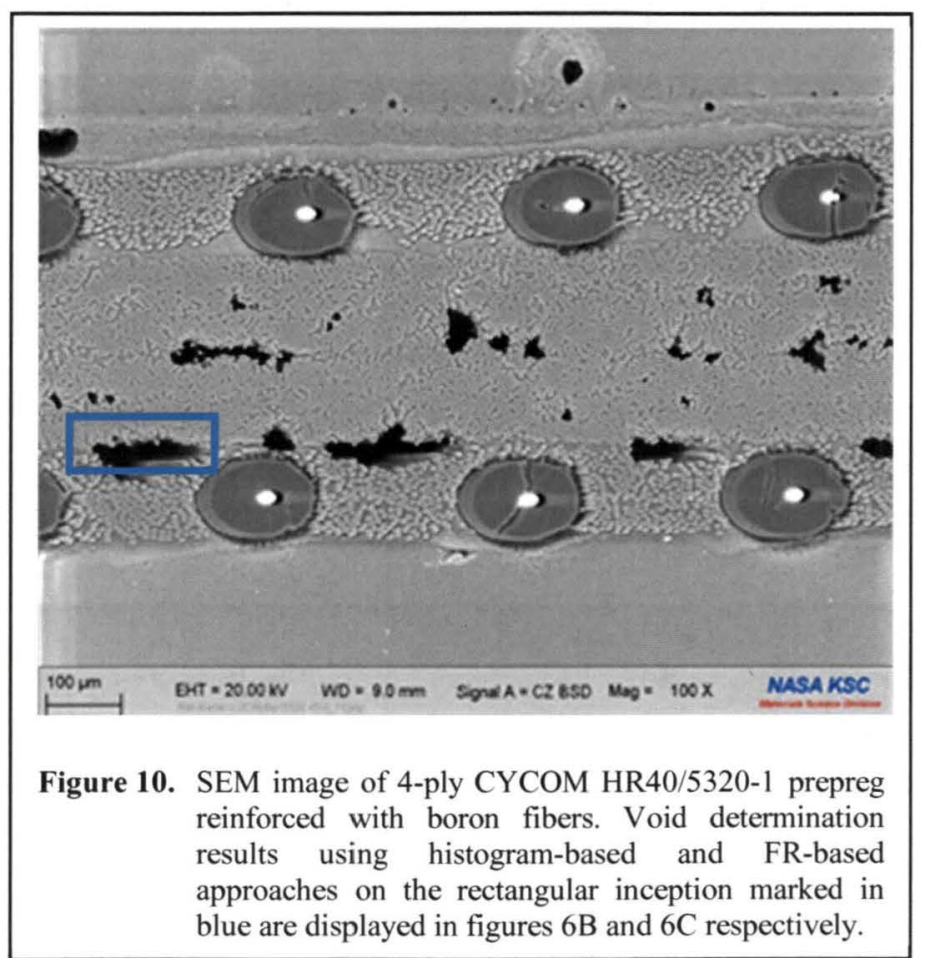

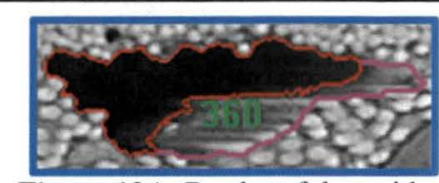

Figure 10A. Border of the void segment generated by binary segmentation is marked in red.

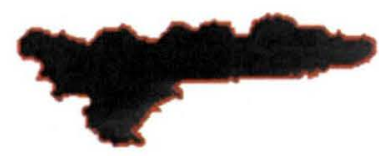

Figure 10B. Void area extracted from Figure 6A for comparison purpose.

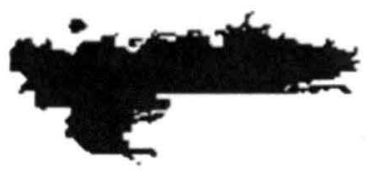

Figure 10C. Void area generated by new FR-based technique.

Accurate void determination on the SEM image shown in figure 10 is particularly challenging despite its first-rate quality and resolution since in some void segments (as the one shown in the blue-marked rectangular inception of figure 10) the interior walls of the voids are not shaded and wrongly taken as continuation of the surface making the gray-scale values of the walls basically identical to those of the surface. Figure 10A shows magnification of the inception taken from figure 10 and marked with a blue-sided rectangle; the borders marked in red are the boundaries of the void area found using manual binary segmentation of the image histogram to choose the optimal threshold value. Figure $10 \mathrm{~B}$ is the void area extracted from figure 10A. Figure 10C is the void area found using the new FR-based binary segmentation via FREIB and FRAT. Comparing figures 10B and 10C leads to the conclusion that even though in both cases the void walls mask a portion of the void segment, the FR-base binary segmentation includes the bottom of the wall as part of the void segment getting a more accurate void area than the manual histogram-based technique. As shown in figure 10A there is an additional void spot located at the right top side that is not detected by the manual histogram-based technique while it is clearly detected in detail by the FR-base binary segmentation as shown in figure $10 \mathrm{C}$. 


\section{CONLUSIONS}

FR approach performs an effective definition of image segmentation and pixel classification on subsequent areas with similar pixel values, such as void and matrix segments within not only optical micrographs but also SEM images of composite samples allowing more accurate image-based determination of void fraction on composite samples. Even with high-quality images, such as optical micrographs and SEM images it is very difficult to differentiate subsequent void-matrix segments due to their similarity in gray-scaled values. FR includes heuristic techniques to identify image attributes. The conventional image techniques are not capable of identifying these image attributes due to their limitation in dealing with the imprecise nature of the digital image acquisition process.

Due to the physical properties of imaging devices and due to the image transmission, images have sometimes poor contrast, are corrupted with different kinds of noise, or are blurred. Image enhancement encompasses different classes of point and spatial operations to increase the contrast, smooth the regions of interest, and sharpen the edges and fine structures. There is large number of techniques for improvement of image quality: gray-level modification, histogram operations, filters based on order statistics, adaptive filters, filters using Fourier/wavelet transform, anisotropic diffusion, morphological filters, genetic algorithms, etc.

Fuzzy set theory is gaining importance in the image processing community in the last decade. Fuzzy techniques are nonlinear, knowledge-based, and robust. They can process imperfect data if this imperfection originates from vagueness and ambiguity rather than randomness. The potentials of fuzzy set theory with respect to image enhancement are still unexplored.

The enhancement approach should be selected in accordance with the ultimate purpose on the outputted image and the quality criterion (or criteria) on the original (input) image. The image processing approach claimed in this paper enhances poor-quality input images by contrasting their image brightness via fuzzy reasoning to overcome vagueness and generate outputted gray-scaled images fitted for the generation of binary images in which the desired segmentation of originally blurred image areas. The binary segmentation of the enhanced image is performed by a technique based also on fuzzy reasoning and developed at Kennedy Space Center and commercially being offered by NASA.

\section{ACKNOWLEDGMENTS}

This work was funded under the NASA Composites for Exploration Project and Composite Cryotank Project and the authors would like to acknowledge Air Force Research Laboratory (AFRL), Defense Advanced Research Projects Agency (DARPA) and Boeing for material and technical consultancy.

\section{REFERENCES}

[1] Patent 7,496,237. Image Processing for Binarization Enhancement via Fuzzy

[2] Patent 7,298,897. Optimal Binarization of Gray-Scaled Digital Images via Fuzzy Reasoning

[3] Dominguez, J. A., Klinko S. Image Analysis Based on Soft Computing and Applied on Space Shuttle Safety during the Liftoff Process. Intelligent Automation and Soft Computing, Vol. 14, No. 3, pages 319-332, 2008

[4] Chi, Z., H. Yan, and T. Pham. Fuzzy algorithm with applications to image processing and pattern recognition advances in fuzzy systems applications and theory, World Scientific Publishing Co., 1999, Vol. 10.

[5] Dominguez, J. A., Klinko S. Intelligent Automation and Soft Computing, Vol. 14, No. 3, pages 319-332, 2008 Visual Anomaly Detection via Soft Computing. Mathware and Soft Computing, Madrid, Spain, 2004, 2-4347 